\title{
Unit commitment by annealing-genetic algorithm
}

\author{
C.-P. Cheng, C.-W. Liu*, C.-C. Liu \\ Department of Electrical Engineering, National Taiwan University, Taipei, Taiwan
}

Received 2 November 1998; revised 18 May 1999; accepted 3 January 2001

\begin{abstract}
This paper presents the application of the annealing-genetics (AG) algorithm to solve the unit commitment (UC) problem. By exploiting the similarity between a minimization process and the cooling of a molten metal, the simulated annealing (SA) guides the search point toward a global minimum with probability 1 using a devised move generation strategy and cooling schedule. But the SA usually takes much computation time in order to arrive at a near-global minimum. The genetic algorithm (GA) is a general-purpose optimization technique based on the principle of natural selection and natural genetics. The GA is a fast algorithm but usually has the inferior solution quality compared to the SA. The AG algorithm incorporates the genetic algorithm into the simulated annealing to improve the performance of the simulated annealing in solving combinatorial optimization problems such as the unit commitment problem. Numerical results on two cases including a realistic Taiwan power (Taipower) system and comparisons with results obtained using the AG, the SA, the GA, the dynamic programming (DP) and the Lagrangian relaxation (LR), show that the features of easy implementation, fast convergence, and highly near-optimal solution to the UC problem can be achieved by the AG. (C) 2002 Elsevier Science Ltd. All rights reserved.
\end{abstract}

Keywords: Unit commitment; Simulated annealing; Genetic algorithm; Annealing-genetic algorithm

\section{Introduction}

The task of the unit commitment (UC) problem involves scheduling the on/off status, as well as the real power outputs, of thermal units for use in meeting the forecasted demand over a future short-term $(24-168 \mathrm{~h})$ period. The resultant schedule should minimize the system production cost during the period while simultaneously satisfying the load demand, the spinning reserve, and physical and operational constraints of the individual units. Since an improved UC schedule may save the electric utilities millions of dollars per year in production costs, the UC problem is an important optimization task in the daily operational planning of modern power systems.

Mathematically, the UC problem may be formulated as a non-linear, large scale, mixed-integer combinatorial optimization problem. The exact solution to the problem can be obtained only by the complete enumeration, often at the cost of prohibitive computational time for realistic power systems [1]. Research endeavours, therefore, have been focused on efficient, near-optimal UC algorithms, which can be applied to large-scale power system and have reasonable storage and computational time requirements. A survey

\footnotetext{
* Corresponding author. Tel.: +886-2-2263-525; fax: +886-2-23638247.

E-mail address: cwliu@cc.ee.ntu.edu.tw (C.-W. Liu).
}

of literature on the UC methods reveals that various numerical optimization techniques have been employed to approach the UC problem. Specifically, there are the priority list method [2,3], the integer programming [4,5], the dynamic programming [6-11], the Lagrangian relaxation method [12,13], the mixed integer programming [14], the branch-and-bound method [15,28], and so on. Among these methods, the priority list method is simple and fast, but the quality of final solution is quite far from the optimum. The dynamic programming method based on a priority list is flexible, but the computational time suffers from 'curse of dimensionality'. The branch-and-bound method adopts a linear function to represent the fuel consumption and time-dependent start cost and obtains the required lower and upper bounds. The shortcoming of the method is that the execution time required increases rapidly for the largescale UC problem. The integer and mixed-integer methods adopt linear programming techniques to solve and check for an integer solution. These methods have only been applied to the small UC problem and have required major assumptions that limit the solution space. The Lagrangian relaxation method provides a fast solution but it suffers from the numerical convergence and the solution quality.

Aside from the above methods, there is another class of numerical techniques applied to the UC problem. Specifically, there are the artificial neural network [16,17], the simulated annealing (SA) [18] and the genetic algorithm 
(GA) $[19,20,23,24,29]$. These methods can accommodate more complicated constraints and are claimed to have better solution quality. The SA is a powerful, general-purpose stochastic optimization technique, which can theoretically converge asymptotically to the global optimum solution with probability 1 . One main drawback of the SA is, however, that it takes a great deal of CPU time to find the near-global minimum. The GA is a general-purpose stochastic and parallel search method based on the mechanics of natural selection and natural genetics. In contrast to the SA, the GA is a fast search method but has inferior solution quality. Recently, Wong and Wong [30] have developed the hybrid GAA2 algorithm for the UC problem. The GAA2 algorithm is based on the combination of the incremental GA approach and the SA technique with population size of 2 to minimize the memory requirement. Empirical results show that the GAA2 algorithm has excellent performance for the UC problem [25-26].

In this paper, we apply an Annealing-Genetics (AG) algorithm to solve the UC problem. The basic idea of the AG is that the GA is incorporated into the SA in such a way that it reduces the computational time of the SA while retaining the main characteristics of the SA, i.e. the ability to achieve the near-global minimum in a finite time. The AG has been successfully applied to many difficult combinatorial optimization problems [22]. A description of the AG algorithm is presented in Section 2. Then a detailed application of the AG algorithm to the UC is given in Section 3. Numerical tests on two cases using the AG, the SA, the GA, the dynamic programming and the Lagragian Relaxation are presented in Section 4. Finally, a summary conclusion is given in Section 5.

\section{Annealing-genetics algorithm}

For the AG algorithm to be efficiency we require (1) the gap between the final solution and the optimal solution should be less than that of the GA and (2) the computational time must be less than that of the SA. These two goals are achieved by designing an annealing schedule that has incorporated the advantages of the GA. The important parameters affecting the efficiency of the SA are the initial temperature, the total length of the Markov chain at each temperature, the equilibrium condition, and the frozen condition. The AG uses the notions from the GA to initiate and control these parameters. A brief description of the $\mathrm{AG}$ algorithm is given in the remainder of this section. A detailed AG algorithm can be found in [22].

The AG algorithm starts with a randomly generated population $P_{0}^{\prime}$. Next, the genetic operators are applied to produce a new population $P_{0}$ by rejecting the higher cost offspring so that the average cost must be less than the random cost $P_{0}^{\prime}$. Then, the Markov chains are generated from $P_{0}$ by a small value of temperature until the next generation $P_{1}$ is created. That is, starting with a random point from $P_{0}$, the next point is generated from the current point by the move generation strategy. An explanation of this strategy applied to the UC problem is given in Section 3.1.5. The initial value of temperature is obtained by dividing the difference between the points of highest cost and the lowest cost of $P_{1}$ by half of the population size, i.e.

Unitial temp $=\frac{(\text { the highest cost }- \text { the lowest cost })}{(\text { the population size/2) }}$

In this way, the acceptance probability of the largest detrimental move will approximately be $0.6(\cong \exp (-\Delta \mathrm{C} / \mathrm{T})$, where $\Delta \mathrm{C}=$ new cost - old cost).

Progressively, for $k=1,2, \ldots$, starting with the point of the lowest cost of $P_{k}$, the next point is generated from the current point by the move generation strategy. If it is accepted by the Metropolis criterion [18], the next point not only becomes the current point but also a point of the quasi-generation $P_{k+1}^{\prime}$; otherwise, a new starting point from $P_{k}$ is attempted. After the population $P_{k+1}^{\prime}$ has been generated at each temperature $T_{k}$, the genetic operators such as crossover, mutation, and reproduction are applied to produce a new population $P_{k+1}$ of the next generation.

An explanation of these genetic operators applied to the UC problem is given in Section 3.1.3. Finally, when more individuals of the population in a certain generation have the same lowest cost, the frozen condition is signaled.

Consequently, the AG algorithm consists of a two-stage cycle. The first stage is the search by the simulated annealing and the second stage is the evolution by the genetic operators. Fig. 1 shows the basic configuration of the AG approach. In each search stage, an iterative generate-andtest procedure is executed to generate many search paths that create a quasi-population. In each evolution stage, a set of genetic operators of the production function is applied to the quasi-population to evaluate their fitness and to produce the population of the next generation. Usually, the production function makes the next generation more mature than the last generation and creates a set of schemata of the population. On the other hand, the schemata are also used to guide the searches of the search stage in the next cycle. They have the power to guide the directions of the search paths toward to high performance regions.

Our AG approach has the following features: (1) it can be viewed as a simulated annealing algorithm with the population-based state transition and with the genetic-operatorbased quasi-equilibrium control, (2) it can be viewed as a genetic algorithm with the Boltzmann-type selection operator.

\section{Application to unit commitment problem}

In this section, we first formulate the UC problem, and then present a detailed AG algorithm for solving the UC problem.

The objective of the UC problem is the minimization of 


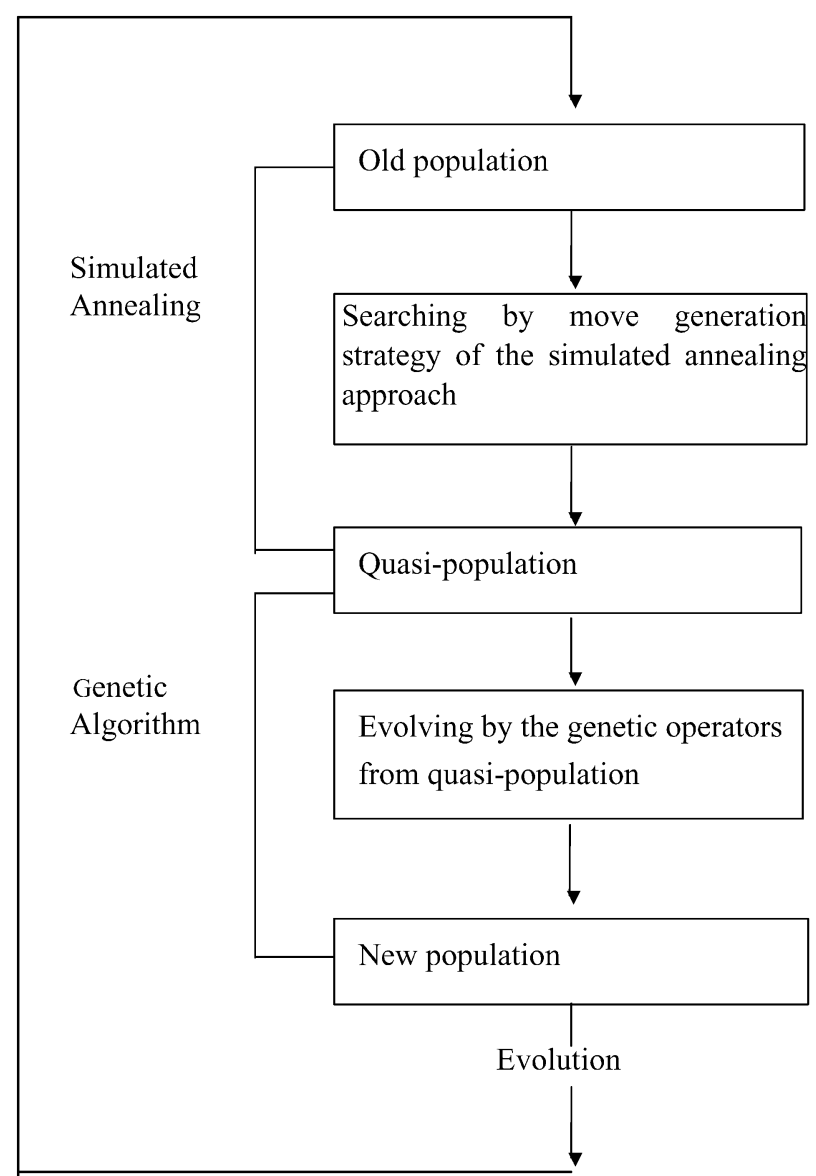

Fig. 1. The basic configuration of the AG algorithm.

the total production costs over the scheduling horizon. Therefore, the objective function is expressed as the sum of fuel and start-up costs of the generating units. Mathematically, the function is as follows:

$C(X, P)=\sum_{i=1}^{N} \sum_{h=1}^{H}\left[X_{i h} F_{i}\left(P_{i h}\right)+S T_{i} X_{i h}\left(1-X_{i(h-1)}\right)\right]$

where

$F_{i}\left(P_{i h}\right)$ : fuel cost function of the $i$-th unit with generation output, $P_{i h}$, at the $h$-th hour. Usually, it is a quadratic polynomial with coefficient $a_{i}, b_{i}$ and $c_{i}$ as follows:

$F_{i}\left(P_{i h}\right)=a_{i} P_{i h}^{2}+b_{i} P_{i h}+c_{i}$

$S T_{i}: \quad$ the start-up cost of the $i$-th unit,

$P_{i h}$ : the generation output of the $i$-th unit at the $h$-th hour,

$P: \quad$ the generation matrix with $P_{i h}, \quad i=1,2, \ldots, \mathrm{N}$; $h=1,2, \ldots, H$, as elements,

$X_{i h}: \quad$ the on/off status of the $i$-th unit at the $h$-th hour, and $X_{i h}=0$ when off; $X_{i h}=1$ when on,

$X: \quad$ the schedule matrix with $X_{i h}, i=1,2, \ldots, \mathrm{N}$; $h=1,2, \ldots \mathrm{H}$, as elements,
$N: \quad$ the number of units,

$H: \quad$ the number of hours.

Now, we consider the constraint equations. Basically, the constraints result from the following consideration of the system operation:

1. balance of power supply and load demand;

2. spinning reserve;

3. generation limit;

4. minimum up-and down-time;

5. ramp rate limit.

Mathematically, these equality and inequality constraints can be expressed in the following vector forms:

$L(X, P)=0$

$G(X, P) \leq 0$

Note that the inequality vector means that every component of the vector is an inequality equation.

In summary, combining Eqs. (1)-(3) yields a complete formulation of the UC problem as the following:

Minimize

$C(X, P)$

Subject to

$L(X, P)=0$

$G(X, P) \leq 0$

Note, there are two matrices $X, P$ to be solved in the above equations to yield the minimum production cost. In this paper, the AG algorithm searches $X$ and $P$ is computed by the Lambda-iteration method [1]. A stepby-step AG algorithm for the UC problem is outlined as follows.

\subsection{The AG algorithm for UC}

Step 1: Initialize the parameters such as the random number seed, the cooling rate $(\alpha)$, the Boltzman factor, the size of population, the mutation rate, the crossover rate, the penalty factor and the number of moves at each temperature;

Step 2: Randomly generate the initial population $P_{0}^{\prime}$;

Step 3: Apply genetic operators to $P_{0}^{\prime}$ to create $P_{0}$;

Step 4: Perform the Lambda_iteration of each decoded point in $P_{0}$;

Calculate the fitness value and the cost of each point in $P_{0}$;

Step 5: Solution_vector: $=$ current_point: $=$ the point of the lowest cost in $P_{0} ; k:=0$;

Step 6: While (the system is not frozen) do

$$
\text { \{no_of_point: }=0 \text {; }
$$


Step 7: While (no_of_point $\leq$ population size) do

\{Generate next_point from current_point using a move generation strategy; check the feasibility of the next-point, and if any constraint is violated then goes to step $7 ; \Delta C:=(\operatorname{cost}$ of next_point) - (cost of current_point);

$$
\begin{aligned}
& P_{r}:=\min \left[1, \exp \left(\frac{-\Delta C}{T_{k}}\right)\right] \\
& \text { if }\left(P_{r}>\text { random }[0,1]\right) \text { then } \\
& \quad\left\{\text { put next_point into } P_{k+1}^{\prime} ;\right. \\
& \quad \text { current_point: }=\text { next_point; } \\
& \quad \text { no_of_point: }=\text { no_of_point }+1 ;\} \\
& \text { else } \\
& \quad \text { \{pick another point from } P_{k} \text { as } \\
& \text { current_point; } \\
& \text { \} end while }
\end{aligned}
$$

Step 8: Apply the genetic operators to $P_{k+1}^{\prime}$ to create $P_{k+1}$; Step 9: Perform the Lambda_iteration of each point in $P_{k+1}$;

Calculate the fitness and the cost of each point in $P_{k+1}$;

Step 10: If (point with the lowest cost in $P_{k+1}^{\prime}<$ solution_vector) then

\{solution_vector: $=$ the point with the lowest cost in $P_{k+1}$; current_point: $=$ the point with the lowest cost in $P_{k+1}$;

Step 11: If (it is the initial stage) then

\{determining the initial temperature $T_{1}$;

$T_{1}:=$ (the highest cost - the lowest costs)/ (the population size/2);

\}

else $\left\{T_{k+1}=T_{k}^{*} \alpha ;\right\}$

Step 12: $k:=k+1$;

Step 13: If (frozen condition is signaled) then \{set system to be frozen;

Step 14: \} end while

Step 15: Print out the solution-vector as the final solution;

Some explanations regarding the AG algorithm are given in the following.

\subsubsection{Explanation 1}

A point in the AG corresponds to an encoded schedule matrix in the UC problem. The matrix form of a point is shown in Fig. 2.

The $i$-th row of the point denoting the states of the $i$-th unit is a binary string divided into many binary substrings with 4 bits each such as $Y_{i 1}, Y_{i 2}, Y_{i 3}, Y_{i 4}$. The first substring, $Y_{i 1}$, of each row represents one operating state of on or off (with a ' 1 ' or ' 0 ' in the leading bit) and the number of hours where on_state (or off_state) lasts for. The other substrings, $Y_{i 2}, Y_{i 3}, Y_{i 4}$, of each row denote one operating state of on or encoded on/off state

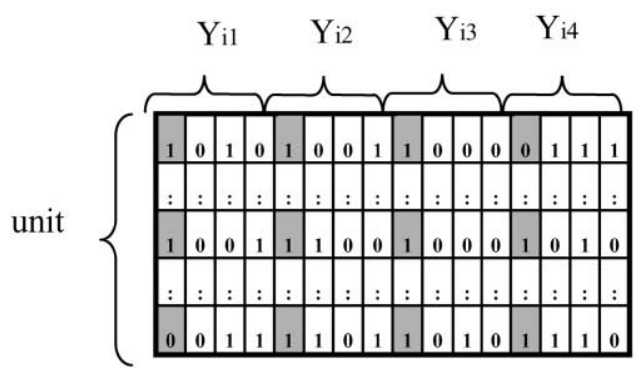

Fig. 2. Matrix form of a point.

off (with a ' 1 ' or ' 0 ' in the leading bit) and the number of hours of the on_state (or off_state) which exceeds the minimum up_time (or down_time). In this manner, the constraints of minimum up-time and down-time can be embedded in the binary substrings, $Y_{i 2}, Y_{i 3}, Y_{i 4}$ and will be satisfied implicitly during the genetic operation to greatly reduce the dimension of search space. If the overall number of hours represented by these substrings is less than the total $H$ hours, then the last substring $Y_{i 4}$ is appended by the operating state, which is the same as the previous state until the $H$-th hour. For example, the $i$-th unit has a minimum uptime of $2 \mathrm{~h}$ and a minimum down-time of $3 \mathrm{~h}$. The actual state of the $i$-th row of the schedule matrix and corresponding encoded $i$-th row of a schedule matrix are shown in Fig. 3.

\subsubsection{Explanation 2}

The fitness function translates the objective function with the constraints into a fitness value. The violations of constraints except the minimum up-time and down-time are penalized by a penalty factor. In the $A G$, the proposed

\section{actual state of the i-th unit}

\begin{tabular}{|l|c|c|c|c|c|c|c|c|c|c|c|c|}
\hline Hour & 1 & 2 & 3 & 4 & 5 & 6 & 7 & 8 & 9 & 10 & 11 & 12 \\
\hline Actual State & 1 & 1 & 1 & 1 & 1 & 0 & 0 & 0 & 0 & 0 & 0 & 0 \\
\hline Hour & 13 & 14 & 15 & 16 & 17 & 18 & 19 & 20 & 21 & 22 & 23 & 24 \\
\hline Actual State & 0 & 0 & 0 & 0 & 0 & 0 & 0 & 1 & 1 & 1 & 1 & 1 \\
\hline
\end{tabular}

encoded i-th row of a schedule matrix

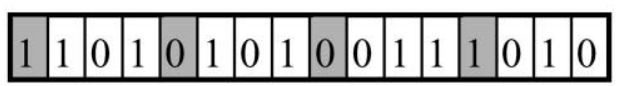

Fig. 3. Actual state and corresponding encoded $i$-th row of a schedule matrix. 
fitness function is expressed as follows:

$$
F(X, P)=\frac{1}{1+K\left(\frac{F_{\max }}{F_{r}}-1\right),}
$$

where

K: $\quad$ a scaling coefficient,

$$
F r:=\frac{1}{C(X, P)+\sum_{h=1}^{H} \alpha_{h} \cdot L},
$$

$C(X, P)$ : the objective function of the UC (the production cost function)

$\alpha_{h}$ : $\quad=1$, when the violation of the constraints exists at the $h$-th hour,

: $\quad=0$, otherwise,

$F_{\text {max }}$ : the maximum of $F_{\mathrm{r}}$ of an individual point within the population,

$L: \quad$ the penalty factor.

\subsubsection{Explanation 3}

There are three genetic operators in the AG, namely reproduction, crossover, and mutation. The reproduction operator is a prime selection operator that copies an old point into a 'mating pool' according to the 'roulette wheel parent selection' technique $[21,27]$. In this technique, the points with larger fitness value have a higher probability to be selected.

The crossover operator recombines the extremely important features of two points to make the offspring points not only inherit some important characteristics from their parent points but also have the chance to get closer to the optimal solution. In the AG, we adopt a new crossover technique known as 'uniform crossover' [27], which exchanges bits between the parent points to create two new offspring points by a randomly generated mask. The scheme of 'uniform crossover' is shown in Fig. 4. In the random mask, the ' 1 ' represents bit swapping and ' 0 ' denotes bit unchanged.

The mutation operator allows us to create new point in the population and provides background variation depending on a mutation probability. The scheme of mutation operator is shown in Fig. 5.

\subsubsection{Explanation 4}

The 'roulette wheel parent selection' technique is used to select the 'better' parent points according to their fitness. It consists of the following steps [21,27]:

Step 1: Sum the fitness of all points in the population; call it the FITSUM.

Step 2: Generate a random number, $P$, between 0 and FITSUM.

Step 3: Return the first chromosome whose fitness, added to the fitness of preceding points, is greater than or equal to $P$.

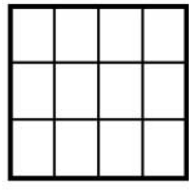

PARENT POINT 1

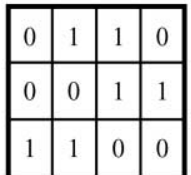

RANDOM MASK

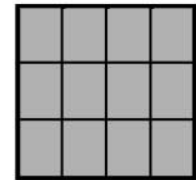

PARENT POINT 2

Uniform crossover

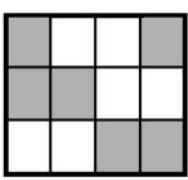

OFF SPRING POINT 2
OFF SPRING POINT 1

Fig. 4. Uniform crossover operator.

\subsubsection{Explanation 5}

The move generation strategy in step 7 of the AG consists of two steps as follows:

Step 1: Randomly pick an element of encoded schedule matrix (a point).

Step 2: Change the state of the element. Eliminate all resulting violations on constraints while making few changes on the commitment of unit in other hours.

\section{Numerical simulations}

In this section, two cases are studied to illustrate the effectiveness of the proposed AG algorithm in terms of its production cost and computational time. The first case compares the AG with deterministic algorithms, i.e. the dynamic programming (DP) and the Lagrangian relaxation (LR). The second case compares the AG with stochastic algorithms, i.e. the simulated annealing (SA) and the genetic algorithm (GA). After testing and evaluating different parameter combinations, the control parameter settings are the scaling coefficient $K=1000$, the penalty factor $L=99999$ and the cooling rate $\mathrm{a}=0.93$. All programs are coded in Turbo $\mathrm{C}$ and implemented on a compatible personal computer (PC 486DX2-66). In order to avoid misleading results due to the stochastic nature of the AG, 20 runs were averaged for each case, with each run starting with different random populations.
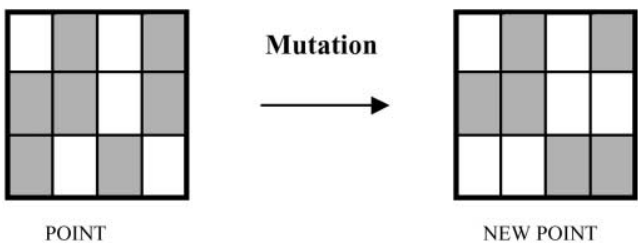

Fig. 5. Mutation operator. 
Table 1

Comparison performance among AG, DP and LR [29]

\begin{tabular}{lccc}
\hline & Cost (\$) by DP & Cost (\$) by LR & Cost (\$) by AG \\
\hline 10 unit & 565,825 & 565,825 & 564,005 \\
20 unit & - & $1,130,660$ & $1,124,651$ \\
40 unit & - & $2,258,503$ & $2,249,072$ \\
\hline
\end{tabular}

\subsection{Case 1: 10-, 20- and 40-unit systems by DP, LR and AG}

In this case, the commitment schedule determined by the proposed AG technique is compared to that determined by the Lagrangian relaxation (LR) and the dynamic programming (DP) [29]. The system unit data and the load demands are shown in Appendix A. After testing and evaluating different parameter combinations, the control parameter settings of AG are the population size 70 , the initial temperature 900, the probability of crossover 0.8 and the probability of mutation 0.1 . The performance of the AG compared with that of LR and DP is given in Table 1 . From this result, it is shown that the solution by AG is better than that of LR and DP in terms of production cost.

\subsection{Case 2: Taipower 40-unit system by SA, GA and $A G$}

In this case, a practical power system, Taiwan Power system (Taipower system), was tested by using the SA, GA and AG. The Taipower system consists of 40 major thermal units. The parameters of operation of units are provided in Appendix B. The utility factors of the thermal system are varied from 72 to $94 \%$ for different seasons of the year to investigate the influences of the load curves on
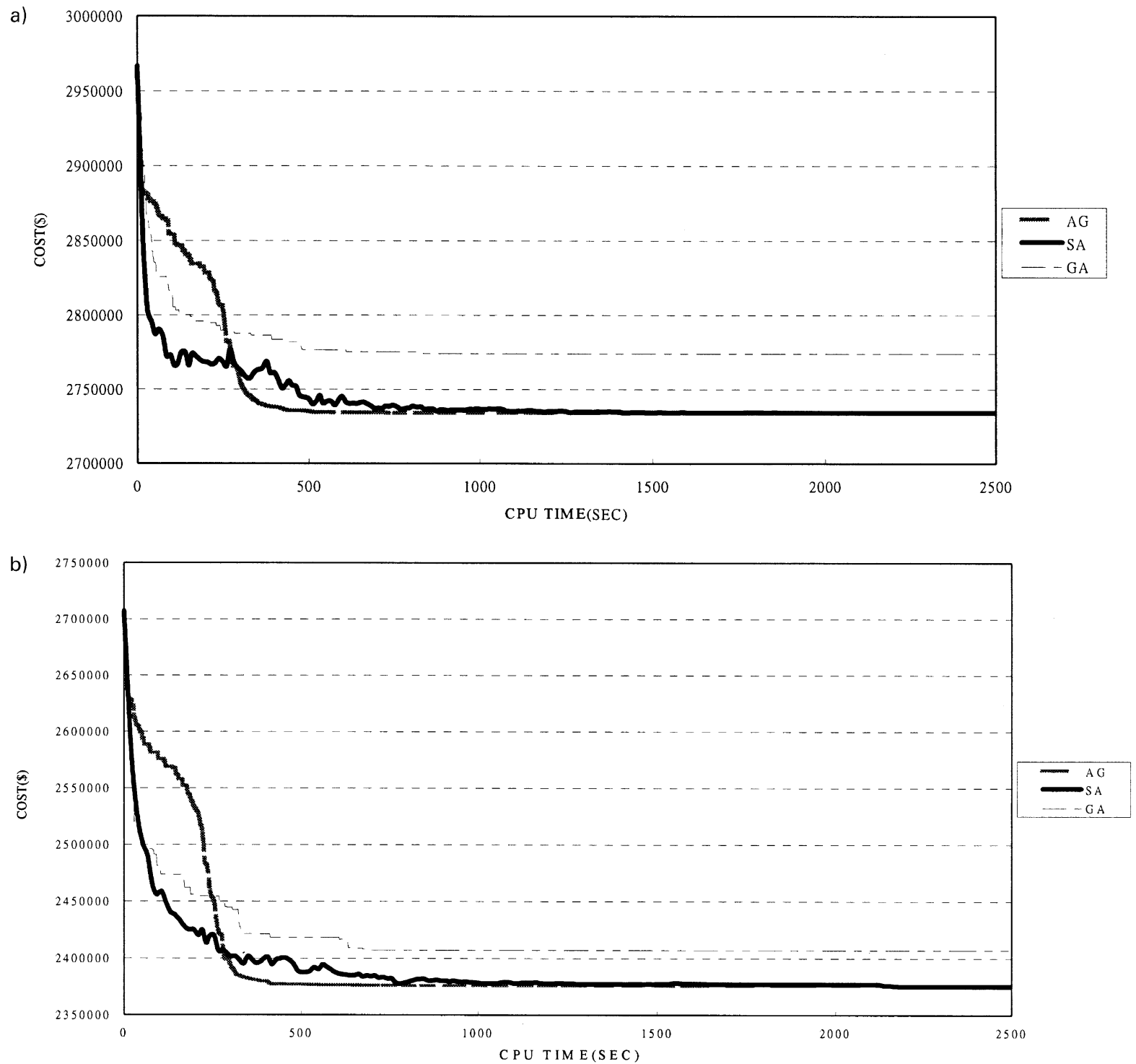

Fig. 6. (a) The search process of AG, SA and GA for $94 \%$ utility factor; (b) The search process of AG, SA and GA for $87 \%$ utility factor; (c) The search process of AG, SA and GA for $80 \%$ utility factor; (d) The search process of AG, SA and GA for $72 \%$ utility factor. 
c)
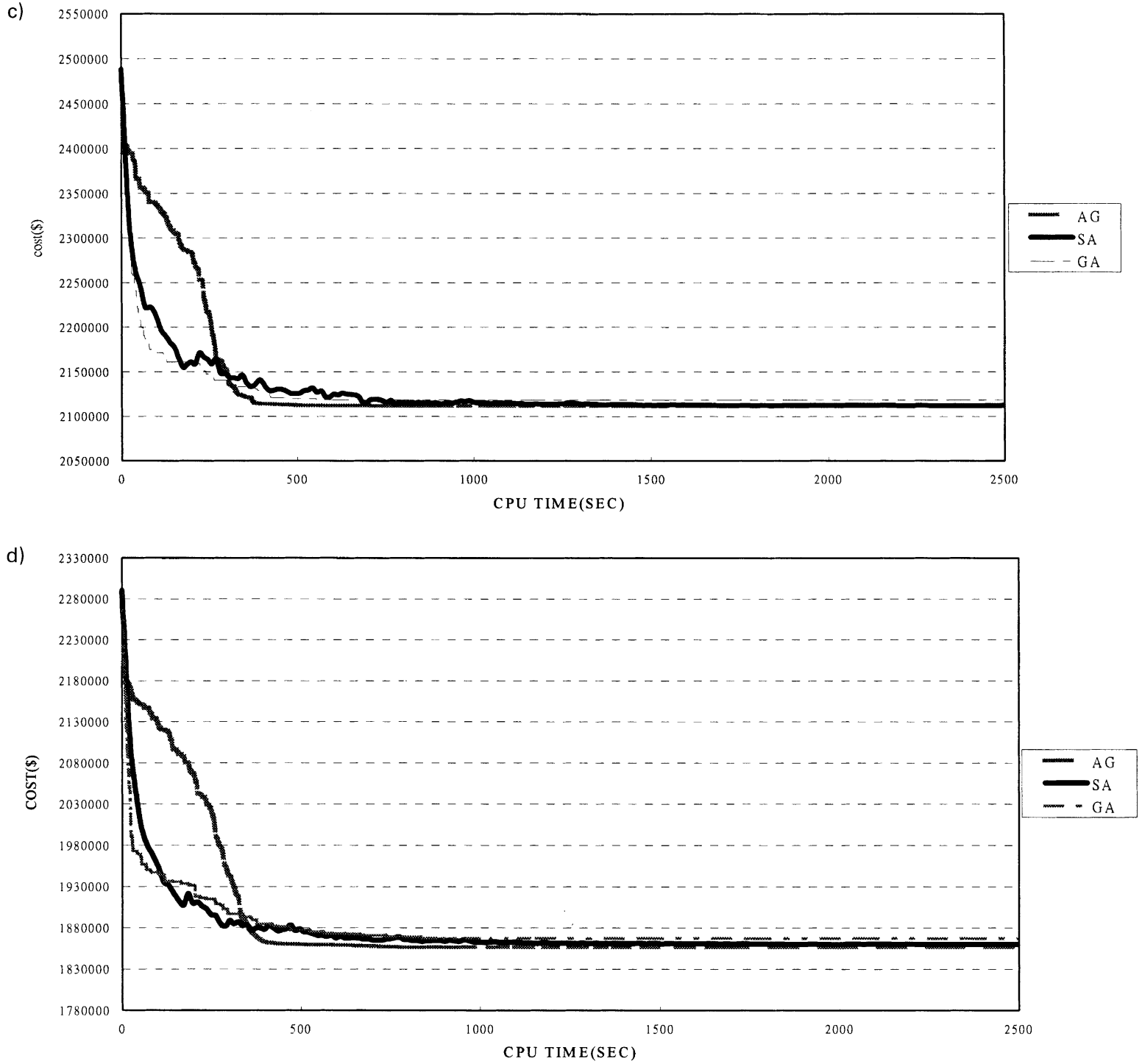

Fig. 6. (continued)

the performance of the AG. The utility factor is defined as

$$
\text { Utility factor }=\frac{\text { Max load }}{\text { Max available system capacity }}
$$

The performance of the AG compared with that of the SA and the GA is given in Fig. 6 and Table 2. From these results, it is shown that the solution by the AG is better than that of the SA in terms of CPU time and better than that of the GA in terms of production cost.

The influences of the various control parameters on the performance of the AG were examined further. We changed one parameter at a time, keeping the other ones unchanged, and evaluated the performance of the AG for the load curve of $94 \%$ in Appendix B. The parameters concerned included the population sizes, and the probabilities of crossover and mutation. The performance for different population sizes is illustrated in Table 3. Effect on the variation of the population size show that a large population requires more evaluations per generation, resulting in a relatively slows rate of convergence; however, a small population retains less variation of the individuals and results in a premature stopping of the AG. The performances for different probability of crossover and mutation are almost same.

\section{Conclusion}

This paper investigates the unit commitment (UC) problem by the Annealing-genetic algorithm (AG) which incorporated the genetic algorithm (GA) into the simulated annealing (SA) algorithm to improve the CPU time of the simulated annealing (SA) algorithm and the solution quality of genetic algorithm (GA). The numerical tests and results 
Table 2

Comparison performance among AG,SA and GA for different utility factor

\begin{tabular}{lllllll}
\hline Utility factor & Cost (\$) by SA & Cost (\$) by GA & Cost (\$) by AG & CPU time (s) by SA & CPU time (s) by GA & CPU time (s) by AG HSAGA \\
\hline $94 \%^{\mathrm{a}}$ & $2,735,427$ & $2,775,344$ & $2,734,402$ & 2402 & 2099 & 1801 \\
$87 \%^{\mathrm{b}}$ & $2,377,755$ & $2,408,643$ & $2,376,098$ & 3092 & 1961 & 2371 \\
$80 \%^{\mathrm{c}}$ & $2,112,288$ & $2,118,544$ & $2,111,830$ & 3954 & 1882 & 2566 \\
$72 \%^{\mathrm{d}}$ & $1,857,257$ & $1,872,381$ & $1,856,584$ & 3313 & 1952 & 3270 \\
\hline
\end{tabular}

a After testing and evaluating different parameter combinations, the control parameter settings are as follows: SA: the initial temperature =900; GA: the population size $=50$; the probability of crossover $=0.6$; the probability of mutation $=0.0333$; AG: the population size $=50$; the probability of crossover $=0.7$; the probability of mutation $=0.1$.

${ }^{\mathrm{b}}$ After testing and evaluating different parameter combinations, the control parameter settings are as follows: SA: the initial temperature $=900$; GA: the population size $=50$; the probability of crossover $=0.8$; the probability of mutation $=0.0333$; AG: the population size $=50$; the probability of crossover $=0.8$; the probability of mutation $=0.3$.

${ }^{c}$ After testing and evaluating different parameter combinations, the control parameter settings are as follows: SA: the initial temperature =900; GA: the population size $=50$; the probability of crossover $=0.8$; the probability of mutation $=0.0333$; AG: the population size $=50$; the probability of crossover $=0.8$; the probability of mutation $=0.1$.

${ }^{d}$ After testing and evaluating different parameter combinations, the control parameter settings are as follows: SA: the initial temperature $=900$; GA: the population size $=50$; the probability of crossover $=0.8$; the probability of mutation $=0.0333$; AG: the population size $=60$; tobability of crossover $=0.8$; tobability of mutation $=0.3$.

show that better solution of the unit commitment (UC) problem can be obtained by the AG algorithm compared with the DP, the LR, the GA and the SA. Moreover, the AG algorithm has the potential to be a practical near-global optimal solution to the UC problem for the large-scale electric power systems.
Table 3

The performance of AG for different population size

\begin{tabular}{lll}
\hline Population size $^{\mathrm{a}}$ & Cost (\$) & CPU time (s) \\
\hline 40 & $2,734,948$ & 1815 \\
50 & $2,734,402$ & 1799 \\
60 & $2,734,643$ & 2481 \\
\hline
\end{tabular}

${ }^{a}$ After testing and evaluating different parameter combinations, the control parameter settings are given in the following: the probability of crossover $=0.8$; the probability of mutation $=0.1$.

\section{Appendix A. Unit data and load demands for case 1 [29]}

Data for the 10-unit system

\begin{tabular}{|c|c|c|c|c|c|c|c|c|c|c|c|}
\hline Unit & $P_{\max }$ & $P_{\min }$ & $a$ & $b$ & $c$ & $\mathrm{Up}$ & Down & $\begin{array}{l}\text { Hot start } \\
\text { cost }(\$)\end{array}$ & $\begin{array}{l}\text { Cold start } \\
\text { cost }(\$)\end{array}$ & $\begin{array}{l}\text { Cold } \\
\text { start (h) }\end{array}$ & it (h) \\
\hline 1 & 455 & 150 & 1000 & 16.19 & 0.00048 & 8 & 8 & 4500 & 9000 & 5 & 8 \\
\hline 2 & 455 & 150 & 970 & 17.26 & 0.00031 & 8 & 8 & 5000 & 10000 & 5 & 8 \\
\hline 3 & 130 & 20 & 700 & 16.60 & 0.002 & 5 & 5 & 550 & 1100 & 4 & -5 \\
\hline 4 & 130 & 20 & 680 & 16.50 & 0.00211 & 5 & 5 & 560 & 1120 & 4 & -5 \\
\hline 5 & 162 & 25 & 450 & 19.70 & 0.00398 & 6 & 6 & 900 & 1800 & 4 & -6 \\
\hline 6 & 80 & 20 & 370 & 22.26 & 0.00712 & 3 & 3 & 170 & 340 & 2 & -3 \\
\hline 7 & 85 & 25 & 480 & 27.74 & 0.0079 & 3 & 3 & 260 & 520 & 2 & -3 \\
\hline 8 & 55 & 10 & 660 & 25.92 & 0.00413 & 1 & 1 & 30 & 60 & 0 & -1 \\
\hline 9 & 55 & 10 & 665 & 27.27 & 0.00222 & 1 & 1 & 30 & 60 & 0 & -1 \\
\hline 10 & 55 & 10 & 670 & 27.79 & 0.00173 & 1 & 1 & 30 & 60 & 0 & -1 \\
\hline
\end{tabular}

unit: the number of the unit,

$P_{\max }, P_{\min }$ : the maximum and minimum power generation,

$a, b, c$ : the coefficients of the fuel cost function,

down, up: the minimum down and up time,

start up cost $=\left\{\begin{array}{l}\text { hot start cost, if down time } \leqq \text { cold start } h \\ \text { cold start cost, otherwise }\end{array}\right.$

it: the initial time of the unit, if it is positive (or negative), indicates the number of hours the unit has been already up (or down). 
The 24 hour load demand

\begin{tabular}{lccc}
\hline Hour & $P_{\text {load }}(\mathrm{MW})$ & Hour & $P_{\text {load }}(\mathrm{MW})$ \\
\hline 1 & 700 & 13 & 1400 \\
2 & 750 & 14 & 1300 \\
3 & 850 & 15 & 1200 \\
4 & 950 & 16 & 1050 \\
5 & 1000 & 7 & 1000 \\
6 & 1100 & 18 & 1100 \\
7 & 1150 & 19 & 1200 \\
8 & 1200 & 20 & 1400 \\
9 & 1300 & 21 & 1300 \\
10 & 1400 & 22 & 1100 \\
11 & 1450 & 23 & 900 \\
12 & 1500 & 24 & 800 \\
\hline
\end{tabular}

The problem data were scaled appropriately for the problems with more units. The reserve was assumed to be $10 \%$ of the demand.

\section{Appendix B. Taipower 40 units' data and load demands for case 2 [27]}

Data for the 40-unit system

\begin{tabular}{|c|c|c|c|c|c|c|c|c|c|}
\hline Unit & $P_{\max }$ & $P_{\min }$ & $a$ & $b$ & $c$ & stc & $\mathrm{Up}$ & Down & it \\
\hline 1 & 80 & 40 & 170.44 & 8.336 & 0.03073 & 247.3962 & 2 & 3 & 3 \\
\hline 2 & 120 & 60 & 309.54 & 7.0706 & 0.02028 & 248.1078 & 2 & 3 & 3 \\
\hline 3 & 190 & 80 & 369.03 & 8.1817 & 0.00942 & 245.8377 & 2 & 3 & 3 \\
\hline 4 & 42 & 24 & 135.48 & 6.9467 & 0.08482 & 246.2440 & 2 & 3 & 3 \\
\hline 5 & 42 & 26 & 135.19 & 6.5595 & 0.09693 & 231.7961 & 2 & 3 & 3 \\
\hline 6 & 140 & 68 & 222.33 & 8.0543 & 0.01142 & 267.5881 & 2 & 3 & 3 \\
\hline 7 & 300 & 110 & 287.71 & 8.0323 & 0.00357 & 267.0076 & 2 & 3 & 3 \\
\hline 8 & 300 & 135 & 391.98 & 6.999 & 0.00492 & 326.6603 & 2 & 3 & 3 \\
\hline 9 & 300 & 135 & 455.76 & 6.602 & 0.00573 & 267.6292 & 2 & 3 & 3 \\
\hline 10 & 300 & 130 & 722.82 & 12.908 & 0.00605 & 376.2845 & 2 & 3 & 3 \\
\hline 11 & 375 & 94 & 635.20 & 12.986 & 0.00515 & 383.9118 & 2 & 3 & 3 \\
\hline 12 & 375 & 94 & 654.69 & 12.796 & 0.00569 & 384.0180 & 2 & 3 & 3 \\
\hline 13 & 500 & 125 & 913.40 & 12.501 & 0.00421 & 384.0245 & 2 & 3 & 3 \\
\hline 14 & 500 & 125 & 1760.4 & 8.8412 & 0.00752 & 380.3792 & 2 & 3 & 3 \\
\hline 15 & 500 & 125 & 1728.3 & 9.1575 & 0.00708 & 386.9936 & 2 & 3 & 3 \\
\hline 16 & 500 & 125 & 1728.3 & 9.1575 & 0.00708 & 380.0032 & 2 & 3 & 3 \\
\hline 17 & 500 & 125 & 1728.3 & 9.1575 & 0.00708 & 247.3962 & 2 & 3 & 3 \\
\hline 18 & 500 & 220 & 647.85 & 7.9691 & 0.00313 & 248.1078 & 2 & 3 & 3 \\
\hline 19 & 500 & 220 & 649.69 & 7.9550 & 0.00313 & 245.8377 & 2 & 3 & 3 \\
\hline 20 & 550 & 242 & 647.83 & 7.9691 & 0.00313 & 246.2440 & 2 & 3 & 3 \\
\hline 21 & 550 & 242 & 647.81 & 7.9691 & 0.00313 & 231.7961 & 2 & 3 & 3 \\
\hline 22 & 550 & 254 & 758.96 & 6.6313 & 0.00298 & 267.5881 & 2 & 3 & 3 \\
\hline 23 & 550 & 254 & 758.96 & 6.6313 & 0.00298 & 267.0076 & 2 & 3 & 3 \\
\hline 24 & 550 & 254 & 794.53 & 6.6611 & 0.00284 & 326.6603 & 2 & 3 & 3 \\
\hline 25 & 550 & 254 & 794.53 & 6.6611 & 0.00284 & 267.6292 & 2 & 3 & 3 \\
\hline 26 & 550 & 254 & 801.32 & 7.1032 & 0.00277 & 376.2845 & 2 & 3 & 3 \\
\hline 27 & 550 & 254 & 801.32 & 7.1032 & 0.00277 & 383.9118 & 2 & 3 & 3 \\
\hline 28 & 150 & 10 & 1055.1 & 3.3353 & 0.52124 & 384.0180 & 2 & 3 & 3 \\
\hline 29 & 150 & 10 & 1055.1 & 3.3353 & 0.52124 & 384.0245 & 2 & 3 & 3 \\
\hline 30 & 150 & 10 & 1055.1 & 3.3353 & 0.52124 & 380.3792 & 2 & 3 & 3 \\
\hline 31 & 70 & 20 & 1207.8 & 13.052 & 0.25098 & 386.9936 & 2 & 3 & 3 \\
\hline 32 & 70 & 20 & 810.79 & 21.887 & 0.16766 & 380.0032 & 2 & 3 & 3 \\
\hline 33 & 70 & 20 & 1247.7 & 10.244 & 0.26350 & 267.6292 & 2 & 3 & 3 \\
\hline 34 & 70 & 20 & 1219.2 & 8.3707 & 0.30575 & 376.2845 & 2 & 3 & 3 \\
\hline 35 & 60 & 18 & 641.43 & 26.258 & 0.18362 & 383.9118 & 2 & 3 & 3 \\
\hline 36 & 60 & 18 & 1112.8 & 9.6956 & 0.32563 & 384.0180 & 2 & 3 & 3 \\
\hline 37 & 60 & 20 & 1044.4 & 7.1633 & 0.33722 & 384.0245 & 2 & 3 & 3 \\
\hline 38 & 60 & 25 & 832.24 & 16.339 & 0.23915 & 380.3792 & 2 & 3 & 3 \\
\hline 39 & 60 & 25 & 834.24 & 16.339 & 0.23915 & 386.9936 & 2 & 3 & 3 \\
\hline 40 & 60 & 25 & 1035.2 & 16.339 & 0.23915 & 380.0032 & 2 & 3 & 3 \\
\hline
\end{tabular}

unit: the number of the unit:

$P_{\max }, P_{\min }:$ the maximum and minimum power generation;

$a, b, c:$ the coefficients of the fuel cost function;

stc: $\quad$ start up cost;

up, down: the minimum up and down time;

it: the initial time of the unit, if it is positive (or negative), indicates the number of hours the unit has been already up (or down). 
Load demand of $94 \%$ utility factor

\begin{tabular}{lcll}
\hline Hour & $P_{\text {load }}(\mathrm{MW})$ & Hour & $P_{\text {load }}(\mathrm{MW})$ \\
\hline 1 & 700 & 13 & 1400 \\
2 & 750 & 14 & 1300 \\
3 & 850 & 15 & 1200 \\
4 & 950 & 16 & 1050 \\
5 & 1000 & 17 & 1000 \\
6 & 1100 & 18 & 1100 \\
7 & 1150 & 19 & 1200 \\
8 & 1200 & 20 & 1400 \\
9 & 1300 & 21 & 1300 \\
10 & 1400 & 22 & 1100 \\
11 & 1450 & 23 & 900 \\
12 & 1500 & 24 & 800
\end{tabular}

The load demand was scaled appropriately for the problems with utility factor.

\section{References}

[1] Wood AJ, Wollenberg BF. Power generation, operation \& control. John Wiley \& Sons, Inc, NY, 1984.

[2] Burns RM, Gibson CA. Optimization of priority lists for a unit commitment program. Paper A 75 453-1, presented at the IEEE/ PES, July 1975 Summer Meeting.

[3] Sheble GB. Solution of the unit commitment problem by the method of unit periods. IEEE Trans on Power Systems 1990;5(1):257-60.

[4] Dillon TS, Edwin KW. Integer programming approach to the problem of optimal unit commitment with probabilistic reserve determination. IEEE Trans on Power Apparatus and Systems 1978;PAS-97(6):215466.

[5] Garver LL. Power generation scheduling by integer programmingdevelopment of theory. IEEE Trans on Power Apparatus and Systems 1963;102:730-5.

[6] Snyder Jr WJ, Powell Jr JD, Rayburn JC. Dynamic programming approach to unit commitment. IEEE Trans on Power Systems 1987;2:339-50.

[7] Lowery PG. Generation unit commitment by dynamic programming. IEEE Trans on Power Apparatus and Systems 1983;102:1218-25.

[8] Pang CK, Chen HC. Optimal short-term thermal unit commitment. IEEE Trans on Power Apparatus and Systems 1976;95(4):1336-46.

[9] Pang CK, Sheble GB, Albuyeh F. Evaluation of dynamic programming based methods and multiple area representation for thermal unit commitments. IEEE Trans on Power Apparatus and Systems 1981;PAS-100(3):1212-8.

[10] Su CC, Hsu YY. Fuzzy dynamic programming: an application to unit commitment. IEEE Trans on Power Systems 1991;6(3):1231-7.

[11] Ouyang Z, Shahidehpour SM. An intelligent dynamic programming for unit commitment application. IEEE Trans on Power Systems 1991;6(3):1203-9.
[12] Merlin A, Sandrin P. A new method for unit commitment at Electricite De France. IEEE Trans on Power Apparatus and Systems 1983;102:1218-25.

[13] Zhuang F, Galiana FD. Towards a more rigorous and practical unit commitment by Lagrangian relaxation. IEEE Trans on Power Systems 1988;3(2):763-72.

[14] Muckstadt JA, Wilson RC. An application of mixed-integer programming duality to scheduling thermal generating systems. IEEE Trans on Power Apparatus and Systems 1968;PAS-87:1968-78.

[15] Cohen AI, Yoshimura M. A branch-and-bound algorithm for unit commitment. IEEE Trans on Power Apparatus and Systems 1983;PAS-102(2):444-51.

[16] Ouyang Z, Shahidehpour SM. A hybrid artificial neural network/ dynamic programming approach to unit commitment. IEEE Trans on Power Systems 1992;7(1):236-42.

[17] Sasaki H, Watabable M, Kubokawa J, Yorino N, Yokoyama R. A solution method of unit commitment by artificial neural networks. IEEE Trans on Power Systems 1992;7(1):974-85.

[18] Zhuang F, Galiana FD. Unit commitment by simulated annealing. IEEE Trans on Power Systems 1990;5(1):311-7.

[19] Dasgupta D, McGregor DR. Thermal unit commitment using genetic algorithms. IEE Proc C, Gener Transm Distrib 1994;141(5):459-65.

[20] Huang CL, Tzeng JS, Yang PC, Yang HT. Implementation of genetic algorithm for unit commitment. 1993 Proceedings of the 14th Symposium on Electrical Power Engineering, Taiwan, ROC, 1993. p. 43946.

[21] Goldberg DE. Genetic algorithms in search, optimization and machine learning. Addison Wesley, MA, 1989.

[22] Lin FT, Kao CY, Hsu CC. Applying the genetic approach to simulated annealing in solving some NP-hard problems. IEEE Trans on Systems, Man, and Cybernetics 1993;23(6):1752-67.

[23] Sheble GB, Maifeld TT. Unit commitment by genetic algorithm and expert system. Electric Power System Research 1994;30:115-21.

[24] Walters DC, Sheble GB. Genetic algorithm solution of economic dispatch with valve point loading. IEEE Trans on Power Systems 1993;8(3):1325-32.

[25] Weng HC, Kao CY. A genetic algorithm approach for the bounded integer programming problem. Master Dissertation, National Taiwan University, 1993.

[26] Lin CY, Kao CY. A genetic algorithm approach for the sequencing problems: the traveling salesman problem and scheduling problem. Master Dissertation, National Taiwan University, 1993.

[27] Chen P-H, Chang H-C. Large-scale economic dispatch by genetic algorithm. IEEE Trans on Power Systems 1995;10(4):1919-25.

[28] Chen C-L, Wang S-C. Branch-and-bound scheduling for thermal generating units. IEEE Trans on Energy Conversion 1993;8(2):184-9.

[29] Kazarlis SA, Bakirtzis AG, Petridis V. A genetic algorithm solution to the unit commitment problem. IEEE Trans on Power Systems 1996;11(1):83-92.

[30] Wong KP, Wong YW. Thermal generator scheduling using hybrid genetic/simulated-annealing approach. IEE Proc C, Gener Transm Distrib 1995; 142(4):372-80. 